This item was submitted to Loughborough's Research Repository by the author.

Items in Figshare are protected by copyright, with all rights reserved, unless otherwise indicated.

\title{
The potential of adaptive interfaces as an accessibility aid for older web
} users

PLEASE CITE THE PUBLISHED VERSION

PUBLISHER

(c) ACM

VERSION

AM (Accepted Manuscript)

LICENCE

CC BY-NC-ND 4.0

\section{REPOSITORY RECORD}

Sloan, David, Matthew T. Atkinson, Colin H.C. Machin, and Yunqiu Li. 2019. "The Potential of Adaptive Interfaces as an Accessibility Aid for Older Web Users". figshare. https://hdl.handle.net/2134/6262. 
This item was submitted to Loughborough's Institutional Repository (https://dspace.lboro.ac.uk/) by the author and is made available under the following Creative Commons Licence conditions.

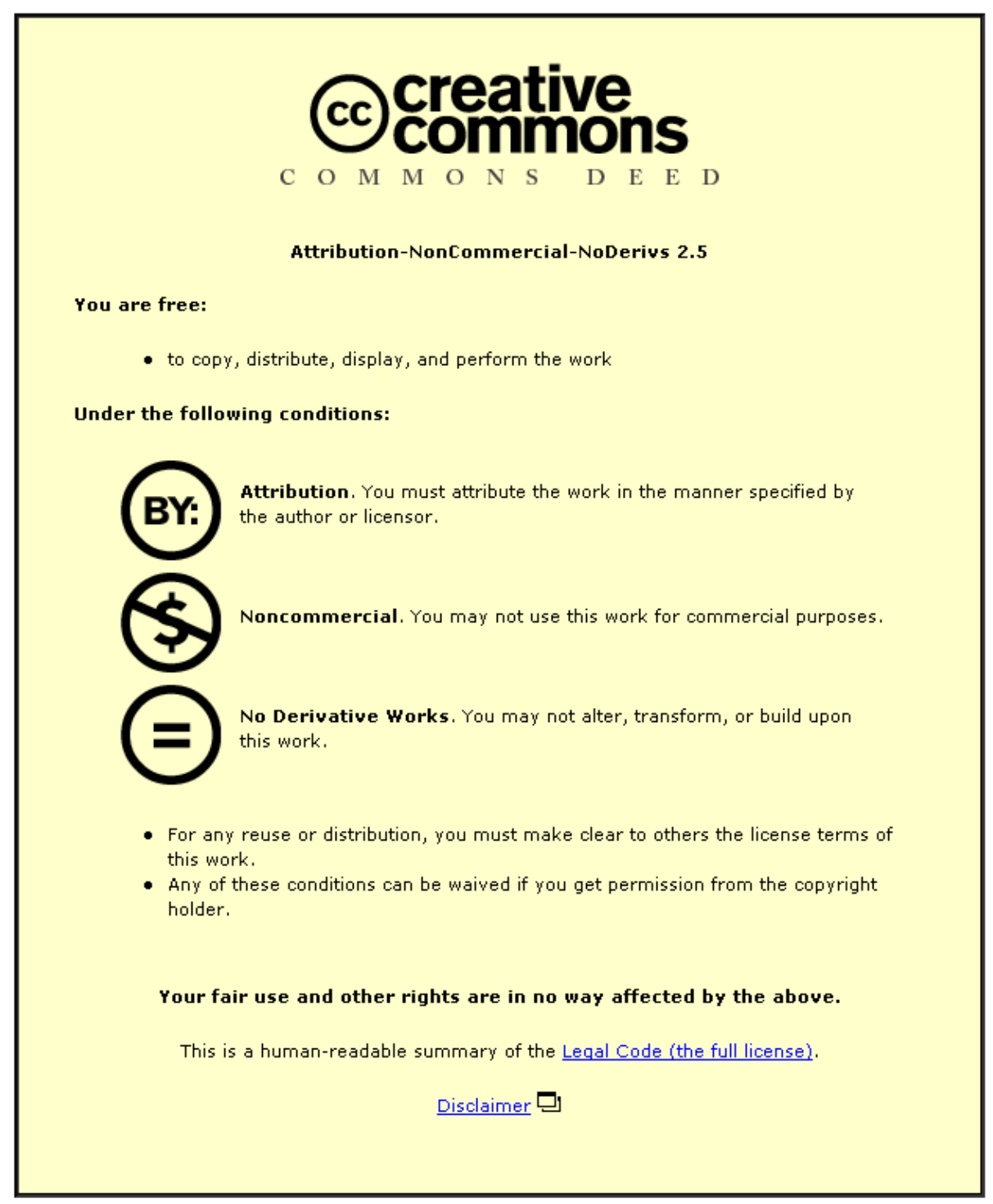

For the full text of this licence, please go to: http://creativecommons.org/licenses/by-nc-nd/2.5/ 


\section{The Potential of Adaptive Interfaces as an Accessibility Aid for Older Web Users}

\author{
David Sloan \\ School of Computing \\ University of Dundee \\ Dundee, UK DD1 4HN, \\ +44 (0)1382385598 \\ dsloan@computing.dundee.ac.uk
}

\author{
Matthew Tylee Atkinson, Colin Machin, \\ Yunqiu Li \\ Department of Computing Sciences \\ Loughborough University \\ Loughborough, UK LE11 3TU \\ +44 (0)1509635658
}

\begin{abstract}
Supporting effective and enjoyable Web usage by people with sensory, motor and cognitive impairments requires more than just accessible Web content. There is an additional task of matching people with an accessibility solution that best accommodates their particular needs - which, especially for older Web users, may fluctuate in severity, number and combination. Lack of awareness of one's own accessibility needs and the solutions that may exist to accommodate them may lead to a reduced quality Web browsing experience or even abandonment.

This paper discusses the difficulties in matching people with less severe, but multiple, impairments with the most appropriate accessibility features at a given time, and explores the role of automated or semi-automated adaptations as a solution for this problem. We review related work, and report on the early stages of our own work conducted to prove the concept of adaptations for accessibility in the specific context of supporting Web users with age-related capability decline. We also consider the potential ethical issues of automated and semi-automated accessibility adaptations on the wellbeing of older Web users, and how these might best be managed in a suitably sensitive way.
\end{abstract}

\section{Categories and Subject Descriptors}

K.4.2 [Computing Milieux]: Computers and Society - Assistive technologies for persons with disabilities. K.3.2 [Computing Milieux]: Computers and Education - Curriculum.

\section{General Terms}

Design, Human Factors, Legal Aspects.

\section{Keywords}

Accessibility, Inclusion, Adaptation, Older People, Web.

\section{INTRODUCTION}

The challenge of improving the experience of disabled people when using the Web has a significant overlap with activities aimed at improving the experience of older people in accessing

\footnotetext{
Permission to make digital or hard copies of all or part of this work for personal or classroom use is granted without fee provided that copies are not made or distributed for profit or commercial advantage and that copies bear this notice and the full citation on the first page. To copy otherwise, or republish, to post on servers or to redistribute to lists, requires prior specific permission and/or a fee.
}

W4A'10 - Technical April 26-27, 2010, Raleigh, North Carolina, USA. Copyright 2010 ACM 978-1-4503-0045-2...\$10.00.
\{M.T.Atkinson,C.H.C.Machin, K.Y.Li\}@lboro.ac.uk and using the Web, many of whom may have, or are likely to experience a decline in sensory, motor or cognitive capability [16][22]. Much progress has been made in improving the accessibility of Web content and applications, and also in the quality and availability of assistive technology that can support disabled people in using technology in general and the Web in particular. For many older people, however, Web usability remains a significant issue. Dynamically changing accessibility needs, resulting from age-related capability decline, mean that current models for supporting accessibility on the Web have serious limitations. In particular there remains a challenge in connecting a person with specific accessibility needs with the right accessibility solution.

In this paper, we investigate the role that automated and semiautomated accessibility adaptations could play in supporting the particular needs of individuals who are experiencing gradual and fluctuating decline in capabilities,. We explore progress in making the Web more accessible, from the perspectives of content production and of assistive technology provision, discuss current limitations with respect to older Web users, and describe our work in investigating adaptations as a potential solution to the problem.

\section{PROGRESS IN MAKING THE WEB MORE ACCESSIBLE}

As the concept of accessibility for disabled and older people matures within the wider sphere of the evolution of the World Wide Web as a unique socio-technological phenomenon, it is now widely acknowledged that for a truly inclusive Web experience to take place, two conditions must be met:

1. Web content must be designed to be as accessible as possible to as many members of the target audience as possible, regardless of any sensory, motor or cognitive impairments they may have.

2. Web users must have, and use, the most appropriate accessibility solution to accommodate their specific accessibility needs.

\subsection{Developments in Web Content Accessibility}

The challenge of promoting and supporting accessible Web content creation is being met on a number of fronts, including:

- The development, by bodies such as $\mathrm{W} 3 \mathrm{C}$ and ISO, of robust international and national standards and sets of guidelines for accessible Web content authoring, covering static content, multimedia, and dynamic interactive Web applications, and the tools used to create such content. 
- Adoption of accessibility needs within broader technical specifications of Web technologies and content formats (for example HTML and PDF).

- The creation and distribution of tools supporting Web authors in evaluation of Web content and addressing of accessibility barriers.

- The emergence of an accessibility industry, supplying the demand for specialist advice on creating accessible and inclusive Web content;

- The promotion of accessibility as part of a standard set of skills that a competent Web design professional should acquire and use, and the provision of resources to support education of content developers in accessibility;

- An active research community exploring ways of enhancing accessibility to disabled people through improved browsers, content design and support for tasks such as navigation and search [17].

An active body of research also exists which is focused on generating evidence-based advice to Web authors on creating usable Web content for older people [2][16]. However, there have been gaps in the relationship between best practice in Web authoring for disability and Web authoring for ageing [34], and bridging these gaps has been a major focus of the W3C's WAIAge project [38].

\subsection{Developments in Assistive Technology}

$N B$ In this section we define assistive technology as an umbrella term to cover any piece of software or hardware that is used to accommodate a sensory, physical or cognitive impairment.

Assistive technology research and development complements activity to promote the creation of accessible Web content by focusing on giving people with accessibility needs of varying levels of severity the appropriate technology to accommodate those needs. The benefits of this work are firstly to take advantage of Web content created with accessibility in mind, and secondly to improve as best as possible the accessibility of Web content that has not been designed with accessibility in mind.

Assistive technology may be present at one or more levels of a Web browsing system architecture:

- Standalone assistive technologies - discrete hardware and software applications that typically provide complex and/or thorough accommodations to significant impairments. For example, a screen reader exists to accommodate a lack of any functional vision; a head mouse exists to accommodate a lack of any use of the hands.

- Assistive technologies are also increasingly integrated at operating system (OS) level, and provide a range of accommodations, in many cases to less severe accessibility barriers, without requiring procurement and installation of specialist standalone assistive technology (see Table 1). This phenomenon exists not just on desktop operating systems but also on mobile platforms.

- At the browser level, accessibility support exists as part of basic browser functionality, including the ability to enlarge text, override display characteristics such as text and background colours, and enhance keyboard interaction. The range, reliability and prominence of features offered ranges across individual browsers.
- Given recent trends towards supporting extension of browser functionality through scripting, for example add-ons and bookmarklets, a number of assistive technologies are available in this way. These typically bring together a range of accessibility options such as display customisation, or basic text-to-speech functionality, and provide them as additional features of the browser interface.

- Finally, there is a category of assistive technology that is provided by the content provider above and beyond the obligations they have as described in Section 2.1. These are typically limited in scope, and may only exist for a single page, group of pages or Web site. Examples might include the provision of text-resising options, alternative style sheets, and text-to-speech functionality.

\begin{tabular}{|l|l|l|l|}
\hline Feature & Mac OS $\boldsymbol{X}$ & GNOME & Windows \\
\hline Screen magnification & $\mathrm{I}$ & $\mathrm{I}$ & $\mathrm{I} / \mathrm{C}$ \\
\hline Colour deficit support & $\mathrm{P}$ & $\mathrm{P}$ & $\mathrm{C}$ \\
\hline Resolution/text size & $\mathrm{P}$ & $\mathrm{I}$ & $\mathrm{I}$ \\
\hline Full screen reader & $\mathrm{I}$ & $\mathrm{I}$ & $\mathrm{I} / \mathrm{F} / \mathrm{C}$ \\
\hline Read specific text & $\mathrm{I}$ & $\mathrm{F}$ & $\mathrm{C}$ \\
\hline On-screen keyboard & $\mathrm{C}$ & $\mathrm{I}$ & $\mathrm{I} / \mathrm{C}$ \\
\hline Voice input & $\mathrm{P}$ & $\mathrm{C}$ & $\mathrm{I}$ \\
\hline
\end{tabular}

Legend: $\mathrm{I}=$ Integrated; $\mathrm{C}=$ Commercial or $\mathrm{F}=$ Free add-on; $\mathrm{P}=$ Partial support.

Table 1: Comparison of accessibility features in common operating systems (latest versions; including bundled applications)

\subsection{Assistive technology for older people}

Many of the technologies described in the previous section, particularly those at the operating system or browser level, could accommodate some of the visual, hearing, mobility and cognitive impairments related to the ageing process.

Beyond general assistive technology, there has been activity that attempts to address the apparent mismatch between general consumer ICT and the particular needs and expectations of older people. Complex and multi-functional systems may present substantial cognitive challenges for older people [8]; additionally, older people have been found to have an exaggerated lack of confidence in using technology [24].

This work has raged from the provision of advice aimed at supporting older people in using technology (for example the ' $\mathrm{Be}$ Grand' Web site ${ }^{1}$ ) to specialist, simplified browsers targeted at older, novice Web users [16], and even 'simplified computers', a number of which are now commercially available (for example Ordissimo $^{2}$ ).

\subsection{Mainstreaming assistive technology}

Contrasting the move towards specialist, separate technologies for older people have been the many instances where specific assistive technologies have been mainstreamed to an extent that they have brought benefits to a wider audience. Examples include

\footnotetext{
1 BeGrand: http://begrand.net/

2 Ordissimo: http://www.ordissimo.com/
} 
predictive text for mobile telephones, cassette tape recorders and display of captions on television [7]. Research by Microsoft and Forrester has demonstrated the wide-reaching benefit of accessibility features of a system [12].

The impact of growing expectations of integrated assistive technology to be present in widely-used consumer devices was illustrated when Apple launched the iPhone without support for two key accessibility needs - text-to-speech and a global minimum font size option. The former rendered the device, relying on touchscreen interaction, unusable by anyone who could not see the screen, while the latter issue caused application developers to have to implement it themselves, even in applications that use the iPhone's native graphical user interface toolkit.

Since the original iPhone model was released in 2007, significant accessibility features have been added to it, in the same way as they have to Mac OS X. These features include full-screen magnification, colour control and a novel gesture-based screen reader - and are clearly aimed at moderate-to-severe sensory impairments, as opposed to minor-to-moderate and intermittent difficulties, which affect more of the population. Illustrating this: despite the large investment in adding assistive technologies, the basic issue of a global variable font size has not been solved.

\section{BARRIERS TO PROGRESS}

Section 2.2 described the many instances of assistive technology that can be used to support people with accessibility needs in using the Web; however, frequently the connection between solution and beneficiary is not made. The successful adoption of assistive technology, particularly by older people with less severe, but multiple impairments, can be adversely affected by a number of issues, some of which are generic across all assistive technology usage scenarios.

Economic issues are most likely to affect access to more complex assistive technology, which may be unaffordable to people who would benefit from it. Another barrier relates to the reluctance of funding agencies to support purchase of a cheaper, mainstream device that could be used as an assistive technology over an expensive and limited function assistive technology [27]. Learnability and usability issues relate to the learning curve that faces new users of assistive technology, relating to its complexity or new interaction modes it introduces. The complexity of technologies such as screen readers, with multiple operation modes and keyboard short cuts, can mean users struggle to use their assistive technology to its full potential. This may cause particular issues for older people, where the assistive technology may become a barrier in itself, and is discussed in more detail in the following section.

In contrast to people with more extreme disabilities, there is a more fundamental challenge to overcome in supporting older people with the most appropriate assistive technology - namely:

1. making a person aware that they have accessibility needs;

2. making them aware that a solution exists to accommodate those needs;

3. and providing them with that solution.

This challenge is likely to be most acutely present in a situation where a person acquires an impairment or impairments gradually over time, and may be exacerbated by a lack of technical knowledge, specifically of computer and Web use (compared to, say, the television).

\subsection{Lack of awareness of access needs}

The gradual and unpredictable nature of age-related capability decline means that an individual may be unaware of, or refuses to acknowledge [5], a decline in sensory, motor or cognitive capability that could be accommodated by a relatively minor adjustment to their computer. Capabilities in decline may also produce unforeseen interference effects [23]. Additional factors, such as the availability of aids such as reading glasses or medication, also lead to changes in capability that may not always be predictable, yet may impact on a user's ability to use a computer - even with assistive technology present. This is the concept of dynamic diversity [15] and can also include large fluctuations over a period of years for people from many different backgrounds.

So the need for an assistive technology may not be established until a point at which an older person may notice - or be noticed having - significant difficulty in performing a basic task such as reading text on screen, or using a mouse to click on a button. If an intervention is delayed excessively, the traumatic change caused by the introduction of an assistive technology that dramatically changes how a person interacts with a computer may lead to rejection of the assistive technology or abandonment of ICT use altogether.

While assistive technology within a system may lie undiscovered, the user may in the meantime have adopted certain strategies to accommodate their capability decline that do not involve making adjustments to the computer, but might increase the awkwardness of the task of using their computer, for example by moving closer to the screen in order to be able to read it, or using a different pair of spectacles. The comparative difficulty older computer users may experience in learning to use a system [8] may also reduce their willingness to search for and make adjustments to their computer [35].

\subsection{Discoverability of assistive technology}

Despite the prevalence of adventitious and multiple impairment, there is a tendency for accessibility activity to focus on disability that is paediatric and extreme - but low incidence - impairment over a much more common situation of milder and multiple impairment [6]. This induces a false assumption amongst accessibility solution developers that the intended beneficiaries and peers, carers or others providing immediate support to them know they have accessibility needs, know there is a solution, and are motivated to look for that solution.

The issue of being unaware of one's own accessibility needs is therefore exacerbated by the obscurity and in some cases lack of support for accessibility features as part of browsers, despite the existence of guidelines ${ }^{3}$ that promote accessibility support in browsers. This has been an impediment to exposing to users features that might benefit from them [35]. Use of an accessibility feature of the operating system, or browser, or browser extension of the sort described in Section 2.2 fundamentally requires awareness of its existence and relevance to the user's specific access needs - and may require someone to remember a series of

\footnotetext{
3 W3C User Agent Accessibility Guidelines (UAAG): http://www.w3.org/TR/WAI-USERAGENT/
} 
steps in order to reactivate it at a later date, which may require excessive cognitive demands.

Even when in-built accessibility features are provided by an operating system, they might be grouped under the banner of "disability access," where terminology or iconography might indicate information only for people with severe impairments [35]. In fact, these built-in tools are often aimed precisely at those who need only incidental help some of the time and thus do not need a full-featured - and expensive - access technology like a wheelchair.

The economic barriers to accessing some assistive technology may have less impact on people with age-related capability decline, but the obscurity problem still exists for open source, and most likely free, assistive technologies which have the potential to support people with a range of impairments. Unfortunately, without concerted marketing efforts, the visibility of these open systems is likely to be even lower than commercial AT products; though projects such as ÆGIS [21] aim to address this through the promotion of and development of open-source ATs. Thus there is a risk that these more flexible and powerful ATs are most likely to be used by disabled technology experts who seek out or demand open source alternatives, rather than those who more urgently need simple and effective solutions.

The issue of findability extends to organizations that can provide support in matching an individual with the appropriate assistive technology. The shortcoming once again is that the connection between the individual and the source of help must be made. For that to happen, the individual must be aware that they are in a position where they would benefit from help.

\subsection{Attitude to assistive technology}

Alongside the issue of connecting an older person to the appropriate assistive technology are issues relating to the impact of assistive technology use on an individual's sense of well-being, independence and confidence. This may be particularly relevant to older people experiencing age-related capability decline, and a perceived loss of independence may lead to assistive technology being rejected [5]. Older people have been shown to prefer less disruptive accessibility solutions to a full-blown assistive technology [23]

Additionally, Sayago and Blat, in studying a group of older Web users, found that many Web-related tasks carried out by members of the group were done collaboratively, involving two or more people sharing one computer [33]. The involvement of an assistive technology, such as a screen magnifier, may reduce - or be perceived as reducing - the usability of the computer for the partner without impairments, adversely affect the social quality of the collaborative activity. Thus the partner requiring the assistive technology may be disinclined to use it in a collaborative situation.

Yet, in exploring where design meets disability, Pullin [30] cites spectacles as an example of where improvements in design quality - and attitudes - has led to an assistive technology being transformed from something that is ugly, socially awkward and stigmatising to one where the assistive technology is a fashion accessory in its own right and therefore no longer stigmatising. The reluctance to use an assistive technology such as spectacles could therefore be reduced if assistive technology use itself was more fully normalised as part of the ageing process.

\section{ADAPTATION FOR ACCESSIBILITY}

Despite advances in the range, quality and availability of assistive technology, there will always be the fundamental challenge of providing a user with the right solution at the right time for their specific accessibility needs. We propose that automated or semiautomated adaptations for accessibility could be a solution to the particular challenges facing older Web users experiencing agerelated capability decline.

\subsection{Adaptation as an accessibility aid}

The concept of adaptive interfaces to support accessibility requirements is not a new one. Adaptation forms a core thread of the 7 Principles of Universal Design [26]; many of the assistive technologies described in Section 2.2 apply adaptations of some form or another in order to accommodate a sensory, motor or cognitive impairment, for example increasing the size of text to support reduced visual acuity or providing audio output of text content to support someone with no functional vision.

Implementation of effective accessibility adaptations requires two key conditions to be fulfilled:

1. Accurate detection of a person's accessibility needs;

2. Appropriate selection and implementation of an adaptation or adaptations that accommodate the needs in as unobtrusive and as helpful a way as possible.

Additionally, given that adaptations are aimed at accommodating gradual changes in capability, they should be implemented as imperceptibly as possible, so as to minimise any traumatic disruption to the way in which a user interacts with their computer. The extent to which this process can be fully automated will depend on the nature of the adaptation.

Adaptations generally exist to detect or mitigate specific user impairments, and target a specific type of interface or platform. Gajos developed abstract user interface (UI) rendering techniques that take some needs of vision- and motor-impaired users into account [13]. Others provide a model for and a means to detect very specific impairments, e.g. certain motor problems [18] or colour deficit [19]. Adaptations may be triggered by the detection of a chronic or temporary user impairment or they may be deployed in response to changing environmental factors. Finally, the configuration of the adaptations and the system controlling them is an important and complex matter. Trade-offs exist between the aggressiveness of the system in employing adaptations and the user's sense of control, as well as issues surrounding the privacy of configuration information, which could be used to infer personal details about - and possibly identifying the user [36].

\subsection{Related work}

There has been relatively little practical work in the area of applying adaptations either system-wide - that is, across a range of applications rather than in one particular application, allowing lessons learnt about the user's needs in one application to be applied to others; or to enable the interfaces of applications to be expressed in a wide range of other modalities.

IBM's Web Adaptation Technology (WAT) [31] was developed to make changes to system accessibility settings based both on detected user problems (such as tremors whilst typing) and at the request of the user. The way accessibility settings in WAT are presented to the user - and applied - brings existing but obscure, 
settings to a bespoke in-browser interface so that users can easily find and change them. Further, and importantly for the user, the settings are applied not just to Web content but the application as well - i.e. a text size increase will affect the browser's menus too. Greasemonkey permits users to write and run browser scripts that affect the presentation and may alter some interaction semantics of Web content; potentially for accessibility-benefits [3].

In a system designed to cope with fluctuating user capabilities, device and environmental constraints, there is a possibility that two conflicting adaptations may be required at the same time. Though sometimes there are solutions to this, such as swapping full-screen magnification for GUI widget enlargement in systems designed for users who are both vision- and motor-impaired ([13], fig. 5.4, p. 113), there has been little work on what the general case - and solution - may be. Some existing systems are designed to provide information and interfaces in an accessible way to users, such as AVANTI [10], which recognises the need for adaptivity both at the UI and content levels. These projects lay a lot of the groundwork for a more general system but are generally quite domain-specific in nature so cannot be applied widely as-is.

Several standards exist for abstract mark-up of user interfaces many based on XML - though only some may be suitable for use in adaptive systems [37]. There are a number of key requirements for such interfaces [28][39], namely the ability to be targeted at a wide range of devices, from desktop computers to washing machines, and delivery contexts; providing different means of accessing commands. Examples include direct access for expert users vs. methods for discovering available commands for novices; the ability to be rendered in a number of modalities, taking away some control over the rendering process from the developer; to be personalisable and mutable at run-time, as circumstances on the part of the user or environment change.

Decision-theoretic interfaces [13] provide a method by which an abstract user-interface is transformed into a concrete interface for rendering. This work also proposes that plug-ins for contemporary development tools should be produced that allow the computer to infer the abstract user-interface specification from the actions of a developer designing the GUI. Currently, however, adopting truly abstract (possibly XML-based) user-interface mark-up would require considerable re-training of developers, so is unlikely to happen until the technology matures and offers further perceived business benefits.

The ÆEIS project, seeks to develop open-source assistive technology, based on a "plug and play" cross-platform architecture, as well as building support for accessibility standards (such as ARIA and Braille support) into existing and emerging interface toolkits [21].

Although the adaptations performed by the systems referenced above have been shown to be effective, they do not indicate general capabilities and, therefore, are not useful for informing a bank of more fundamental adaptations. The vast majority of existing work is very specific; looking at particular impairments in particular conditions.

\subsection{User Profiling}

A system that presents customised interaction methods optimised for accessibility will require some form of user profiling system, which maintains an accurate representation of an individual's accessibility needs in a way that supports reusability and portability.

There has been activity in profiling accessibility information, including work to standardise the extent of accessibility information, what should be stored and how. This has been driven by the e-learning community, and in particular work of the IMS AccessForAll metadata initiative. The result of this work has led to the creation of a three-part standard, ISO 24751. This standard, covering accessibility profiling of a learner and accessibility profiling of an electronic learning object, describes how these two sets of metadata might be used together in a learning management system to deliver appropriately adapted resources to suit an individual's needs. While ISO 24751 provides a useful reference in terms of accessibility profiling, its scope does not extend to methods of initial data population, nor of maintenance of accurate user profile information over time.

For an automated or semi-automated adaptation system, it cannot be assumed that the content a user wishes to access has any meaningful accessibility information associated with it, and therefore adaptations must rely on measurements of user capabilities. Additionally, capability measurement should only take place if there is a meaningful and positive adaptation that can be applied to accommodate a detected change in capability.

There are also standard models of human capabilities [11], including some data on how these capabilities are affected by disabilities [4]. However there is currently a dearth of detailed and long-term studies covering the needs of people who may experience minor to moderate impairments (sometimes many simultaneously), and the effects of dynamic diversity on assistive technology effectiveness.

A number of possible systems for storing and maintaining users' preferences have been proposed [13][31][36], which in turn uses one of a myriad of different machine-learning algorithms. There will also be a user model, possibly quite specific to the adaptation being developed [4] [18] [19]. However, the use of profiles or models of humans requires care. There have been objections to the notion of using models and guidelines to test systems, rather than users. This can result in lower awareness of the design issues with developers [29]. We note such objections - especially to the apparent abstraction inversion that is occupying the limited power of a computer with the intractable task of simulating the human user. Such a generic simulation should be unnecessary for an adaptation system that targets individuals experiencing minor-tomoderate impairments, and who in some cases can communicate to the computer that an adaptation is required, specify the type of adaptation required or give feedback - acceptance or rejection regarding adaptations that were system-initiated. For example, zooming into a word-processor document indicates that the user is having difficulty reading the text.

Human capabilities are an ideal starting point for profiling users, as these are universal and portable across a multitude of present and future devices. Work such as the WHO International Classification of Functioning, Disability and Health (ICF) enumerates the capabilities of the human species, which can be used as a starting point for (or superset of) user profiles. However, providing a bridge between capabilities expressed in these standard, human, forms to the specific adaptations and parameters discussed in standards such as ISO 24751 (text zoom, text-to-speech) and device capabilities expressed in the likes of 
$\mathrm{CC} / \mathrm{PP}^{4}$ (such as screen resolution) presents a formidable challenge - one which is beyond the scope of this paper, but is being tackled by the authors.

There are many types of user and disability - and, consequently, user models. This even applies to models within a particular type of disability, such as motor control [20]. Even with standard modelling approaches, such as the Model Human Processor, there can be many different concrete implementations, to be used in different circumstances and for different disabilities [4].

This leads to the issue of bootstrapping - arriving at a reasonable starting profile and set of adaptations for a given user [14][31][36], and an optimal method of gathering this information is a key area of investigation for any accessibility adaptation system. A suitable set of initial questions given to the user may be very helpful in determining which adaptations and more specific detection routines may be required, although any direct request from the user for information at a point when they may be engaged in a separate task will introduce issues of trust and usability.

While the learner profiling work that led to ISO 24751 took place in a domain where there was a natural entrance point at which initial profiling information could be gathered - i.e. registration or matriculation on a specific educational programme, there is less of such an opportunity when considering older technology users. Until the ecosystem of adaptivity is established, social support will likely be needed with the practical problem of getting users up-and-running with an accessibility adaptation system, such as the installation of software components - even if this process is designed to be as accessible as possible. However, this step should be needed only until both users and developers have an expectation of adaptivity in the products and services they use.

\subsection{Ethical Issues}

Accessibility adaptations require an ethically-aware approach to accessibility [1]. The requirement that a system for accessibility adaptations accurately detects a change in capability means that potentially very sensitive data is gathered. This information, if available to an individual may adversely affect their personal sense of well-being and of independence, which in turn may negate the quality of life benefits provided by prolonged use of the Web supported by accessibility adaptations. Additionally, over time, a system that captures information about dexterity, cognitive capability and visual acuity might store data that could indicate a separate health issue unconnected with general age-related capability decline, or indicate that a person may no longer be safe to conduct activities such as driving a car. Should such an accessibility adaptation system have a responsibility to report this data to the user, and/or to other concerned parties?

With sensitivity of data come security issues; and therefore an accessibility adaptation system that attempts to maintain an accurate profile of the capability of a user must do so in a way that shields the data from unauthorised access or use.

\footnotetext{
4 W3C Composite Capabilities/Preferences Profile: http://www.w3.org/Mobile/CCPP/
}

\section{INVESTIGATING FEASIBILITY OF ADAPTATIONS FOR OLDER WEB USERS}

The authors are investigating the feasibility of addressing the particular challenges of supporting Web accessibility for older people as part of the multidisciplinary Sus-IT project, funded under the UK Research Councils' New Dynamics of Ageing (NDA) research programme. The project goal is to explore how ICTs, including the Web, can be used to sustain an appropriate level of independence for older people. Within this more general goal is a specific challenge of sustaining independent access to and use of ICTs by older people - and, by extension, to limit the potential for involuntary abandonment due to age-related capability decline.

As part of the work of Sus-IT, the authors are exploring the feasibility of accessibility adaptations as a way of allowing older people to continue to use the Web and other ICTs even as agerelated capability decline may lead to reduced visual, hearing, dexterity and cognitive processing capability. The aim of this work is to be compatible with both current (legacy) and future (likely abstract, or at least more mutable) systems - adaptations that can be targeted towards devices, whole applications, or widgets, depending on the specificity afforded by the platform.

\subsection{Technical outline}

Our goal is to address the limits of existing adaptation systems by developing techniques for monitoring capability change on a more general level, and to see this work applied in mainstream technology. However this adoption is unlikely to happen until there is a standard way to monitor capability change and request the services of adaptations.

The system under development follows a basic proposal that the operating system should include a lightweight library that is transparently linked to all applications to allow them to communicate with individual adaptations. These adaptations may be interface renderers, allowing widgets to be presented in a range of modalities, or input handlers that may perform operations such as key de-bouncing. As discussed in Section 4.3 there already exist a wide range of preference and machine-learning systems that could be used and enhanced to track user preferences over time and across different applications. Given a suitable problem detection framework, difficulties that the user experiences could be tracked in a similar way, to allow future decisions on adaptations to be more accurately calculated.

Each individual adaptation "plug-in" would be very simple; capable of only one style of rendering - such as text-to-speech and would have the ability to take parameters controlling the adaptation. A controller process, provided with the OS/adaptivity library, would respond to predicted or detected problems the user faces, as well as environmental constraints. Multiple adaptations would be usable concurrently on a given interface, or parts of it. So, for example, a user whose eyes tire when reading a large amount may have certain types of content spoken aloud but toolbars and menus simply enlarged. As much effort as possible should be made to keep preferences system-wide. 


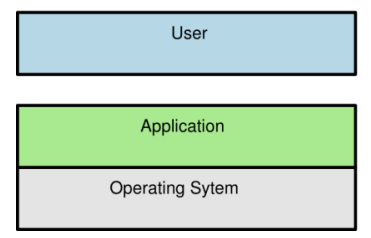

User interacts with application.

Figure 1: Architecture of a current system with no AT installed

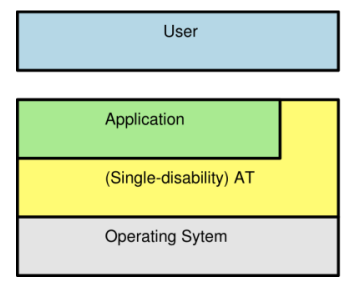

User has a sense of interacting with the application and the AT

OS provides infrastructure for AT to communicate out-of-process to some applications so that their interface controls may be rendered accessibly.

AT installed inside OS to intercept data from inaccessible applications (e.g. those using custom Ul controls).

Figure 2: Current monolithic AT arrangement

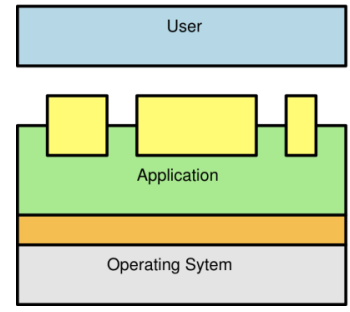

User feels like they are only interacting with the application.

Combinations of adaptations, each focussed on particular functional aspect, are used to render the application's interface and accept input from the user.

OS provides a thin layer for instantiating adaptations that is transparent to applications.

Figure 3: Proposed adaptation-based system.

Figure 1, Figure 2 and Figure 3 illustrate how such an approach may be structured when implemented and how it compares to current systems, both with and without AT. It should be noted that the lightweight adaptation framework could initially be developed separately from, and later installed into, the operating system, though it would ideally be included in the operating system in the long-term.

For legacy applications whose UIs are immutable, only devicelevel adaptations, such as screen magnification, or OS-level adjustments, such as global font size, would be effective. The more mutable an application's UI, the more it may be adapted. Our system would provide a very general, high-level classification of expected user behaviour for an application, such as Windows Media Player or Calculator, so that we can see if user behaviour unexpectedly changes. An example of this might be providing much more input than expected, or switching to keyboard after historically having used keyboard and mouse as input devices. The problem of the myriad specific - and complex - models may be sidestepped if it is considered unnecessary to simulate all possible disabilities a user may have. Instead, what is required is to arrive at a reasonable set of baseline capabilities and then monitor for changes in these capabilities. We monitor capability change on a number of dimensions: (a) an initial assessment which might involve asking users to perform simple tasks that allow assessment of e.g. mouse dexterity; (b) passively monitoring any adaptations or behaviours the user makes in the OS or applications that could signify capability changes (e.g. changing font sizes or contrast) and (c) identification and management of side-effects of adaptations inferred by several means, such as user acceptance or rejection of the adaptations deployed by the system.
A variable range of adaptations, tailored for specific accessibility problems may exist and be present on the user's system. These will specify a number of "trigger" conditions that, when fired, may cause the adaptation itself to ask the user a question or perform some small diagnostic test. If it is found that, for example, the user sometimes experiences a particular type of motor tremor, then that adaptation can be engaged when a drop in overall motor capability is detected and recorded mouse movement data allow the adaptation to verify the problem. This drop in capability might be indicated by the user disengaging from the mouse unexpectedly, after having previously used it to interact with a particular application on the system.

Work is also ongoing to create a technical implementation that can reason about users' changing capabilities - and, importantly, to collect feedback that will help in the evaluation of individual adaptations, the methods of application of those adaptations and the concept of adaptive accessibility as a whole.

Longer-term, more integrated and usable abstract interface specification tools must be created, so that developers are more likely to use such tools to give their products flexible interfaces. A two-pronged approach may be used for this: creating tools for developers that promote the creation of accessible UIs (such as those proposed in the literature, discussed above); also tools may be developed to help patch, at least semi-automatically, existing applications in order to make their interfaces more flexible.

\subsection{Early User Engagement}

Acknowledging the ethical and social issues outlined in Section 4.4, we have adopted a user-centred focus to this work, ensuring that at all stages of the development of accessibility adaptation technology, end user opinion and input captured and used to influence development.

\subsubsection{Theatre as a means of exploring attitudes to accessibility adaptation}

The first stage of our work was to demonstrate the concept of accessibility adaptation to a group of older Web users in order to gauge reaction to the technology, and to instigate a discussion on potential influence on feelings of well-being and security. We used theatre as a way of engaging end users with the concept of accessibility adaptations - this method having been shown to have potential in enhancing the participatory nature of design involving older people and hence improving the quality of the requirements gathering process [25].

A producer was commissioned to work with the project team to develop a series of three dramatic scripts, each focusing on the interaction between two older people and a fictitious simplified home computer, which included accessibility adaptation functionality. Two professional actors were commissioned to perform these scenarios in front of an audience of older people (all aged $60+$ ). The performance lasted approximately 3 hours, including discussion time and a 20 minute break half-way through the performance. The performance was held twice, firstly in front of an audience $(n=16)$ who had little to no experience of using a PC and secondly with an audience $(n=16)$ with more PC usage experience.

During each performance, the producer acted as facilitator, and at strategic moments stopped the performance in order to discuss with the audiences aspects of the technology's behaviour they had just witnessed. Members of the research team were also present, 
as note-takers and to ask additional questions. Both performances were video-recorded.

\subsubsection{Representing accessibility adaptations in a dramatic performance}

The system used as a stage prop for the performance was an LCD flat panel monitor with a cardboard surround. A separate PC, located off-stage and controlled by a member of the research team, was used to simulate the display of the system by projecting PowerPoint slides onto a wall above the stage; additional audio output was simulated by off-stage voice. This gave significant scope to simulate a variety of accessibility adaptations without requiring to implement complex technical solutions.

Presentation of accessibility adaptations within a wider scenario exposing the audience to a simplified home computer did, however, require some careful thought. The project's hypothesis is that the most effective accessibility adaptations are those that automatically and accurately detect changes in an individual's capabilities, and respond in as helpful, and yet in as imperceptible and unobtrusive a way as possible. Given that an individual's capabilities are likely to change very gradually, it would be impractical to present an accurate representation of how we envisage an adaptation system to work in a theatre event limited to a couple of hours in length.

So, the effect of adaptations was deliberately exaggerated, in order firstly to make them noticeable to the audience, and secondly to explore the reaction to a much more extreme instance of an adaptation than would be developed in reality. The adaptations presented to the audience as part of the scenarios were:

- A prominent slider mechanism located below the monitor, which could be physically manipulated by the user to change the size of the text or introduce voice output.

- A system that automatically detected a deterioration in the eyesight of the user, and enlarged the display text until the user pressed a button to stop the increase in size. The adaptation was accompanied by an audio announcement that the user's eyesight had been detected as having deteriorated: "Your eyesight seems to be $11 \%$ worse today, compared with a week ago.. The text size will increase. When you can read the text, press button..."

These exaggerated examples of accessibility adaptations and the characters' reaction to them were woven into the script; and the accessibility adaptations were used as a specific discussion point with the audience.

\subsubsection{Findings}

\subsubsection{Less experienced users.}

The group with less ICT experience reacted positively to the slider enlarging text and button for text to speech. There was empathy with the situation of 'losing my glasses', and no immediate adverse reaction to the adaptations. One observer commented that the textto-speech facility:

"would be a fantastic machine for people who are lonely."

In the second adaptation scenario, again there was no expressed negative reaction to this, although one participant noted the effect of being informed of a change in personal capability:

"it will make me get my eyes tested more regularly!"
The automated aspect of the adaptation was debated - it was generally seen as a "good thing", although several participants felt it would be more appropriate to be alerted in advance and approving a change before an adaptation is made.

In discussing the role of adaptations in making adjustments to the way the system displayed information, mention was made of the instructions provided when the user bought or was given the device. However there was uncertainty within the audience over whether system users would remember this advice over time.

\subsubsection{More experienced users}

As with the less experienced audience, the participants with more ICT experience also reacted positively to the slider-based text resizing feature. As an input mechanism, it was compared favourably to drag-and-drop, even though the latter would be unlikely to be an appropriate interaction style used for text resizing.

There was also a positive reception to the automated text resize adaptation, but with criticisms of the demonstrated implementation. In general participants did not want the system to change without them being alerted, or given the choice. One participant compared this to the now defunct 'Clippy' help feature of Microsoft Word. The issue of trust and data security was also raised - participants queried whether the system would be able to accurately diagnose an accessibility need and make appropriate changes. One participant asked if the data would be sent to his opticians who would then try to sell him new glasses!

\section{DISCUSSION}

The theatre event provided a valuable early exposure of the concept of accessibility adaptations to the target audience. Even considering the potential that the positive reaction may partly be due to a tendency of older people to provide an overly optimistic and uncritical reaction to a technology presented to them to evaluate [9], this may be mitigated by the fact that the concepts presented to them were deliberately exaggerated in order to provoke discussion. Some issues identified in advance were confirmed as being key to user acceptability, while other issues emerged that had not previously been considered.

In addition to the technical work described in Section 5.1, future work will also be influenced by the need to address the following:

- Consideration of an appropriate level of user approval of a specific adaptation before it is applied. The idea that adaptations would be gradually and regularly made with the minimum of disruption, was difficult to convey in the theatre event, so they seemed more invasive than would be intended in reality. There is therefore a balance to be struck between avoiding an unduly invasive adaptation system where a user has to approve every adaptation made, and a system whereby well-meaning but unhelpful adaptations cannot be reversed.

- Accuracy of measurements - this is important, not only in terms of appropriateness of the accessibility adaptation made in response, but also in terms of any information on capability change that is made available to the user.

- Reporting of any capability change that had been detected. Participants did not seem unduly upset by the system reporting a decline in capability, even though this was one aspect of the adaptation system that was deliberately exaggerated. Nevertheless the extent to which, if any, the 
system should make data available to the user about changes in capability is important.

- Data security - users must be confident that any data gathered is stored securely and used only for authorised purposes. The issue of how to deal with data that might have medical implications - for example data on visual acuity that might indicate an underlying health issue - did not emerge during discussions, but clearly is one that remains critical to implementation.

Interestingly, both users groups tended to view the system as potentially useful for 'someone else' older and less capable than themselves - underlining the point that assistive technology is typically not perceived as a helpful feature of a system but rather as something that becomes necessary only in some future lifescenario.

Ongoing and regular user involvement with groups of older ICT users will guide the development of the adaptation system in ensuring that the issues emerging from the initial exposure to the target audience. Beyond this, longitudinal usage studies are planned in order to provide empirical data to tune existing models, and also to explore the extent to which the system can help to sustain independent access to and use of ICT even after significant change in capability.

\section{CONCLUSIONS}

Current accessibility solutions - accessible content, assistive technology and information about accessibility - do not consider people who are experiencing gradual and fluctuating capability change. Overcoming these barriers will require an approach that assumes that users are not aware of their accessibility needs or the most appropriate solution for them. Accessibility adaptations provide potential for an unobtrusive but helpful modification of content display and interaction modes, across different applications and domains, including the Web.

We describe a technique currently under development and testing to bring interfaces and the ATs that adapt these closer together. Ongoing work into more fine-grained capability and adaptation reasoning - allowing adaptations to be applied more specifically to smaller areas of the content or interface (even rendering individual content fragments or widgets in other modalities or on other devices, if required) are under development - will require the coarser approach proposed above to be proven before they are likely to be adopted by developers.

Early exposures of accessibility adaptations to the target audience indicate an appreciation of the benefits such adaptations could provide. At the same time there are substantial technical and social challenges to be met in order to produce a system that supports and sustains older people in using ICT to help optimise quality of life.

\section{ACKNOWLEDGEMENTS}

This work was conducted as part of the Sus-IT project, funded under the UK Joint Research Councils' New Dynamics of Ageing initiative.

We are indebted to Maggie Morgan, David Goodall, Iain Wotherspoon and Jane Nelson Peebles for their roles in producing and performing the theatre, and we thank all those who participated as audience members in the theatre event. We also thank our project colleagues David Frohlich, Sarah Woods, Paula
Forbes and Lorna Gibson, for their efforts in organising the event and in subsequent data analysis and reflection.

\section{REFERENCES}

[1]. Abascal, J. and Nicolle, C. (2005) Moving towards inclusive design guidelines for socially and ethically aware HCI. Interacting with Computers 17 (5). Elsevier, 484-505.

[2]. Arch, A. (2009) Web accessibility for older users: successes and opportunities. Proceedings of 2009 International CrossDisciplinary Conference on Web Accessibility (W4A) Madrid, Spain. New York: ACM Press, 1-6.

[3]. Bigham J., Brudvik, J., Leung, J. and Ladner R. (2009) Enabling web users and developers to script accessibility with Accessmonkey. Disability and Rehabilitation: Assistive Technology 4(4). Informa Healthcare, 288-299.

[4]. Biswas, P. and Robinson, P. (2008) Automatic evaluation of assistive interfaces. In: Proceedings of the ACM International Conference on Intelligent User Interfaces (IUI) 2008. 247-256.

[5]. Blythe, M., Monk, A. and Doughty, K. (2005) Socially dependable design: the challenge of ageing populations for HCI. Interacting with Computers 17 (6). Elsevier, 672-689.

[6]. Carey K. (2005) Accessibility: The Current Situation and New Directions. Ariadne 44, June 2005. Retrieved January29 $9^{\text {th }} 2010:$ http://www.ariadne.ac.uk/issue44/carey/

[7]. Clarkson, P. J., Keates, S., (2003) Countering Design Exclusion: An introduction to inclusive design. SpringerVerlag UK.

[8]. Czaja, S. and Lee, C. C. (2007). The impact of aging on access to technology. Univ Access Inf Soc 5 (4). SpringerVerlag, 341-349.

[9]. Eisma, R., Dickinson, A., Goodman, J., Syme, A., Tiwari, L. and Newell, A. (2004) Early user involvement in the development of information technology-related products for older people. Universal Access in the Information Society 3(2). Springer, 131-140.

[10]. Fink, J., Kobsa, A. and Nill, A. (1998) Adaptable and adaptive information provision for all users, including disabled and elderly people. New Review of Hypermedia and Multimedia 4, 163-188.

[11]. Fleishman, E. A., Quaintance, M. K. and Broedling, L. A., (1984) Taxonomies of human performance: The description of human tasks. Orlando: Academic Press.

[12]. Forrester Research \& Microsoft Corporation (2003). New Research Study Shows 57 Percent of Adult Computer Users Can Benefit From Accessible Technology. Retrieved $1^{\text {st }}$ February 2010 from http://www.microsoft.com/presspass/press/2004/feb04/0202AdultUserBenefitsPR.mspx

[13]. Gajos, K. Z., Wobbrock, J. O. and Weld, D. S.(2007). Automatically generating user interfaces adapted to users' motor and vision capabilities. In: UIST '07: Proceedings of the 20th annual ACM symposium on User interface software and technology. New York: ACM Press, 231-240.

[14]. Gajos, K. Z. (2008) Automatically generating personalized user interfaces. Ph.D. thesis, University of Washington. Retrieved $1^{\text {st }}$ February 2010 from 
http://www.eecs.harvard.edu/ kgajos/papers/2008/kgajosdissertation.pdf

[15]. Gregor, P., Newell, A. F. and Zajicek, M. (2002) Designing for dynamic diversity: interfaces for older people. In: ASSETS '02: Proceedings of the fifth international ACM conference on Assistive technologies. ACM, New York, NY, USA, 151-156.

[16]. Hanson, V. (2009) Age and web access: the next generation. Proceedings of 2009 International Cross-Disciplinary Conference on Web Accessibility (W4A) Madrid, Spain. New York: ACM Press, 7-15.

[17]. Harper, S. and Yesilada, Y. (2008) Web Accessibility - A foundation for research. London: Springer-Verlag.

[18]. Hurst, A., Hudson, S. E., Mankoff, J. and Trewin, S. (2008) Automatically detecting pointing performance. In: Proceedings of the ACM International Conference on Intelligent User Interfaces (IUI) 2008. 11-19.

[19]. Jefferson, L. and Harvey, R. (2007) An interface to support color blind computer users. In: $\mathrm{CHI}$ '07: Proceedings of the SIGCHI conference on Human factors in computing systems. New York: ACM Press, 1535-1538.

[20]. Keates, S., Langdon, P., Clarkson, P. J. and Robinson, P., (2002) User models and user physical capability. User Modeling and User-Adapted Interaction 12 (2-3), 139-169.

[21]. Korn, P., Bekiaris, E. and Gemou, M (2009) Towards open access accessibility everywhere: The ÆGIS concept. Lecture Notes In Computer Science, vol. 5614. Berlin, Heidelberg: Springer-Verlag, , 535-543.

[22]. Kurniawan, S. (2008) Underrstanding Disabilities: Ageing. In: Harper, S. and Yesilada, Y. (eds) Web Accessibility - A foundation for research. London: Springer-Verlag, 47-60.

[23]. Kurniawan, S. H., King, A., Evans, D. G., and Blenkhorn, P. L. (2006). Personalising web page presentation for older people. Interact. Comput., 18(3). Elsevier,:457-477.

[24]. Marquie, J., Jourdan-Boddaert, L. and Huet, N. (2002) Do older adults underestimate their actual computer knowledge? Behaviour \& Information Technology 21 (4). Taylor \& Francis, 273-280.

[25]. Newell, A., Carmichael, A., Morgan, M. and Dickinson, A. (2006) The use of theatre in requirements gathering and usability studies. Interacting with Computers 18 (5). Elsevier, 996-1010.

[26]. NCSU Center for Universal Design (1997). The Principles of Universal Design, Version 2.0. Raleigh, NC: North Carolina State University. Retrieved $1^{\text {st }}$ February from http://www.design.ncsu.edu/cud/about_ud/udprinciples.htm

[27]. New York Times (2009) Insurers Fight Speech-Impairment Remedy. September $14^{\text {th }} 2009$. Retrieved $17^{\text {th }}$ March 2010 from http://www.nytimes.com/2009/09/15/technology/15speech.h tml?_r=4

[28]. Nichols, J., Myers, B., Harris, T., Rosenfeld, R., Shriver, S., Higgins, M. and Hughes, J. (2002) Requirements for automatically generating multi-modal interfaces for complex appliances. Proceedings of Fourth IEEE International Conference on Multimodal Interfaces, 2002., 377-382.

[29]. Petrie, H., Hamilton, F., King, N. and Pavan, P.(2006). Remote usability evaluations with disabled people. In: $\mathrm{CHI}$ '06: Proceedings of the SIGCHI conference on Human Factors in computing systems. New York: ACM Press, 1133-1141.

[30]. Pullin, G. (2009) Design meets Disability. MIT Press.

[31]. Richards, J. and Hanson, V. (2004). Web accessibility: a broader view. In: WWW '04: Proceedings of the 13th international conference on World Wide Web. New York: ACM Press, 72-79.

[32]. Rodrìguez, M., Pérez, J. and Ruìz, M. (2004) Designing user interfaces tailored to the current user's requirements in real time. Computers Helping People with Special Needs, 69-75.

[33]. Sayago, S. and Blat, J. (2010) Telling the story of older people emailing: an ethnographical study. Int J HumanComputer Studies 68 (1). Duluth: Academic Press, 105-120.

[34]. Sloan, D. (2006) Two cultures? The disconnect between the Web standards movement and research based Web design guidelines for older people. Gerontechnology Journal 5(2) (July 2006). 106-112.

[35]. Sloan, D., Dickinson, A., McIlroy, N. and Gibson, L. (2006) Evaluating the Usability of Online Accessibility Information. Final Project Report. Retrieved January2 $9^{\text {th }}$ 2010: http://www.techdis.ac.uk/index.php?p=3_10_10_1.

[36]. Trewin, S. (2000) Configuration agents, control and privacy. In: CUU '00: Proceedings of 2000 Conference on Universal Usability. New York: ACM Press, 9-16.

[37]. Trewin, S., Zimmermann, G. and Vanderheiden, G. (2003). Abstract user interface representations: how well do they support universal access? In: CUU '03: Proceedings of the 2003 Conference on Universal usability. ACM, New York, NY, USA, pp. 77-84.

[38]. W3C (2008) Web Accessibility for Older Users: A Literature Review. W3C Working Draft, 14 May 2008. http://www.w3.org/TR/wai-age-literature/

[39]. Zimmermann, G. and Vanderheiden, G. (2002). Technical requirements for a delivery context independent user interface model. In: W3C Workshop on Device independent authoring techniques. Retrieved $1^{\text {st }}$ February 2010 from http://www.w3.org/2002/07/DIAT/posn/trace.html 